\title{
Where are the boundaries? Automated pocket detection for druggability studies
}

\author{
Andrea Volkamer ${ }^{1 *}$, T Grombacher $^{2}$, Matthias Rarey ${ }^{1}$ \\ From 5th German Conference on Cheminformatics: 23. CIC-Workshop \\ Goslar, Germany. 8-10 November 2009
}

Computer-based prediction of protein druggability is an essential task in the drug development process. Early identification of disease modifying targets that can be modulated by low-molecular weight compounds can help to speed up and reduce costs in drug discovery. Recently, first methods have been presented performing a druggability estimation solely based on the 3D structure of the protein [1-3]. The essential first step for such methods is the identification of the active site. A multitude of methods exist for automated active site prediction [4-6]. However, most methods developed for automated docking procedures do not explicitly focus on the definition of the boundary of the active site. Since druggability estimates are based on structural descriptors of the active site, a precise description of the active site boundaries is vital for correct predictions.

In this work, we present a method to predict protein binding pockets and split them into subpockets such that small molecules are mostly contained within one sub-pocket. The method is based on a novel strategy to geometrically detect narrow regions in pockets. For druggability predictions, such pocket descriptions result in more meaningful structural descriptors like active site surface or volume. Moreover, if several structures from one protein are known, sub-pockets can give hints about protein flexibility and induced fit conformational changes.

Our method was evaluated on 718 proteins from the PDBbind [7] data set, as well as 5419 proteins from the scPDB [8] data set. Binding pockets are correctly predicted in $94 \%$ and $93 \%$ of the datasets. $38 \%$, respectively $45 \%$ of the proteins from the two datasets contain pockets which can be divided into more than one sub-pocket. In all cases one sub-pocket completely covers the co-crystallized ligand. Besides the classical

${ }^{1}$ University of Hamburg, Center for Bioinformatics, Bundesstr. 43, 20146 Hamburg, Germany overlap-measure of ligand versus predicted active site, we additionally considered the pocket coverage by the co-crystallized ligand. We found that the number of test cases with more than $30 \%$ pocket coverage rises from $30 \%$ to $74 \%$ (PDBbind) and from $28 \%$ to $63 \%$ (scPDB), respectively, when considering sub-pockets.

\section{Author details \\ ${ }^{1}$ University of Hamburg, Center for Bioinformatics, Bundesstr. 43, 20146 Hamburg, Germany. ${ }^{2}$ Bioinformatics, Merck Serono, Frankfurter Str. 250, 64293 Darmstadt, Germany.}

Published: 4 May 2010

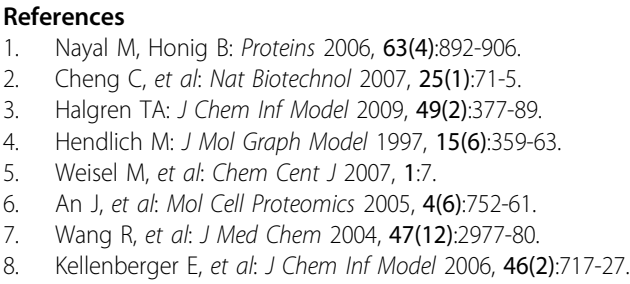

\section{Publish with ChemistryCentral and every} scientist can read your work free of charge

"Open access provides opportunities to our colleagues in other parts of the globe, by allowing anyone to view the content free of charge."

W. Jeffery Hurst, The Hershey Company.

- available free of charge to the entire scientific community

- peer reviewed and published immediately upon acceptance

- cited in PubMed and archived on PubMed Central

- yours - you keep the copyright

Submit your manuscript here:

http://www.chemistrycentral.com/manuscript/

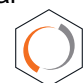
Chemistry Central 